\title{
Crystal-Meth als Herausforderung für das Suchthilfesystem - Klinische Empfehlungen
}

\section{Clinical Recommendations to Meet the Different Needs of Users of Crystal-Meth}

\author{
Autor \\ R. Härtel-Petri \\ Institut \\ FA Psychiatrie und Psychotherapie, Bayreuth
}

\section{Schlüsselwörter \\ - Substitution \\ - Amphetamin \\ - Methamphetamin \\ - Psychostimulantien \\ - Designerdrogen}

Key words

- dextroamphetamine

- methamphetamine

- Germany

- substitution

- replacement therapy

- novel psychoactive substances

- central nervous system stimulants

\section{Zusammenfassung \\ $\nabla$}

Auch Deutschland ist mittlerweile von der Verdrängung des d-Amphetamines durch das $\mathrm{N}$-Methamphetaminhydrochlorid in seiner hochkonzentrierten Form „Crystal-Meth“ in bestimmten Drogenszenen betroffen. Es ist mit einer vermehrten Behandlungsnachfrage zu rechnen.

Diese Übersichtsarbeit fasst die Erfahrungen anderer Länder, auch mit der Amphetaminsubstitution, zusammen.

\section{Einleitung}

\section{$\nabla$}

Die Methamphetaminabhängigkeit gilt als ein weltweites Problem mit hohen sozialen Folgekosten. Nach Cannabis ist Methamphetamin die weltweit am häufigsten konsumierte illegale Droge [1]. Über die Synthese wurde 1893 in Japan von Nagayoshi berichtet, ab 1919 war die Herstellung bereits als kristallines Methamphetamin durch Reduktion des Ephedrins mit rotem Phosphor und Jod in industriellem Maßstab möglich

\section{Bibliografie}

DOI http://dx.doi.org/ 10.1055/s-0035-1559657

Online-Publikation: 28.9.2015

Suchttherapie 2016; 17: 10-16

(c) Georg Thieme Verlag KG

Stuttgart · New York

ISSN 1439-9903

Korrespondenzadresse Dr. med Roland Härtel-Petri FA Psychiatrie und Psychotherapie

Luitpoldplatz 10

95444 Bayreuth

info@psychotherapie-haertelpetri.de
[2]. In Deutschland wurde es von Temmler ab 1923 als Medikament Pervitin ${ }^{\circledR}$ in Dosierungen von $3 \mathrm{mg}$ bis $30 \mathrm{mg} /$ Tag Maximaldosis vertrieben.

\section{Epidemiologie}

Der von den vereinten Nationen früh bemerkte weltweite Trend zu den „Amphetaminartigen Substanzen (ATS)“ [1] zeigte sich in Deutschland zunächst in den Statistiken der Polizei zu den erstauffälligen Konsumenten. Bereits seit 2003 waren die Amphetamine für mehr als 50\% der erstauffälligen Konsumenten verantwortlich [1]. Dabei war und ist die Hauptsubstanz bundesweit

\section{Abstract \\ $\nabla$}

As seen in other countries, dextroamphetamin is replaced by the $\mathrm{N}$-methamphetaminehydrochloride in the almost pure form "Crystal Meth" in drugscenes favouring psychostimulants in Germany. With increasing seizures of methamphetamine and its precursors by German drug enforcement institutions, Germany is confronted with a lack of epidemiological data on abuse and little scientific work on therapeutic approaches. Preventive and therapeutic measures have to be adapted from other countries to this phenomenon emerging over the last two decades. Amphetamine replacement therapy might be one option.

bisher das Dextroamphetamin, das klassische „Speed“ ( $\bullet$ Abb. 1).

Die Hinweise aus Suchtsurveys zur Lebenszeitprävalenzen der Amphetaminartigen Substanzen (ATS) blieben diskret. In der Altersgruppe der heute 40-49 jährigen beträgt die Lebenszeitprävalenz für Amphetamineinnahme 2,1\%. Dies ist die Generation, die in ihren 20igern in der frühen Technoszene mit dem XTC (damals MDMA) in Berührung kam, „DER“ Partydroge, die bis 1986 zunächst noch ein „legal high“ war und klassisches Speed in den gleichen Szenen zur Verfügung stand. Bei den heute 21 bis 29- jährigen beträgt die Lebenszeitprävalenz für Amphetamineinnahme bereits 4,5 bzw. 6,8\% [3].

Diese Zunahme ab der Jahrtausendwende könnte auch in dem Wandel der Konsumgewohnheiten in der ursprünglichen Techno-Rave-Szene liegen. Vom oral eingenommenen niedrig dosierten XTC, sowie den Speedpillen, ging es über einen, als „normal“ erlebten nasalen Amphetaminkonsum mit stärkerer Euphorie und höherem Abhängigkeitspotential, zum kristallinen Methamphetaminkonsum. Kristallines Methamphetamin gilt als schneller zu einem abhängigen Konsum führend, mit nachfolgend vermehrten psychiatrischen Komplikationen [4,5]. 
Zunehmende Behandlungsnachfragen wurden z.B. aus den Suchthilfeeinrichtungen Sachsens berichtet [6] ( 0 Abb. 2). Es war in den späten 90er Jahren bereits auf das, in den Grenzregionen seit ca. 1995 endemische kristalline Methamphetamin, hingewiesen worden [7]. Bis ca. 2009 blieb das kristalline Methamphetamin "Crystal-Meth“ noch weitgehend auf die an die Tschechische Republik angrenzenden Landesteile Deutschlands und Österreichs beschränkt. Ab 2009 war der Markt für die Straßensubstanz „Crystal“, „C“, „Meth“, „Crystal-Speed“, „Piko“ in der Tschechischen Republik von abgeschotteten asiatischen Gruppen übernommen worden. Diese Gruppen hatten ansonsten auf den sogenannten Asiamärkten hinter der Deutsch-Tschechischen Grenze, traditionell unbehelligt, geschmuggelte Zigaretten, gefälschte Uhren, Taschen usw. vertrieben. Seit 2010 wird von Polizei und Zoll von vermehrten Einfuhrversuchen auch größerer Mengen aus der tschechischen Republik berichtet. Nach Polizeiangaben sei der Reinheitsgrad seitdem stark gestiegen, die Preise von früher um 100.- $€ /$ Gramm auf 15 .- $€$ verfallen.

In einer Untersuchung des IFT München [8] in Suchtberatungsstellen in den betroffenen Bereichen entlang der Grenze zur Tschechischen Republik war bei 17\% der Konsumenten das Crystal-Meth die erste illegalisierte Substanz vor Cannabis. Jüngste Funde von Rohstoffen wie APAAN in Drogenlaboren in Deutschland deuten auf Produzenten und Abnehmer im Westen Deutschlands hin, sowie Zeitungsnachrichten auf die Beteiligung der Neonaziszene [9-11].

\section{Wirkung}

Wie das klassische d-Amphetamin unterdrückt das Methamphetamin als „Weckamin“ die Müdigkeit und löst eine Euphorie mit gesteigertem Selbstwertgefühl aus. Bei erhöhtem Rede- und Bewegungsdrang sowie reduzierten sozialen Ängsten erleben sich die Konsumenten kontaktfreudig und sexuell angeregt. Bei vermindertem Schmerz- und Hungergefühl machen „alle Tätigkeiten Spaß“. Es kommt zu stereotypen Verhalten „Punding“ mit zwanghaft anmutendem Schrauben sortieren, Mitesser ausdrücken oder Putzen. Bei Verlust des Zeitgefühls können derartige Tätigkeiten stunden- bis tagelang durchgeführt werden.

Als eher unerwünschte Wirkung kommt es zu einer gereizten Getriebenheit mit reduzierter Konzentrationsfähigkeit und Gedankendrängen sowie bei Überdosierung oder chronischem Konsum zu paranoid psychotischem Erleben. Der erhöhte Muskeltonus der Rückenmuskulatur führt zu Rückenschmerzen, durch die Tonuserhöhung der Gesichtsmuskulatur kommt es zu einem Grimassieren („Gesichtsfasching“) und Zähneknirschen (Bruxismus). Der Nebeneffekt einer Gewichtsabnahme wird anfangs häufig begrüßt. Hypertherme Krisen mit Rhabdomyolyse sind beschrieben. Durch die Hypertonie und Tachycardie kommt es zu cardialen Komplikationen sowie Apoplexien.
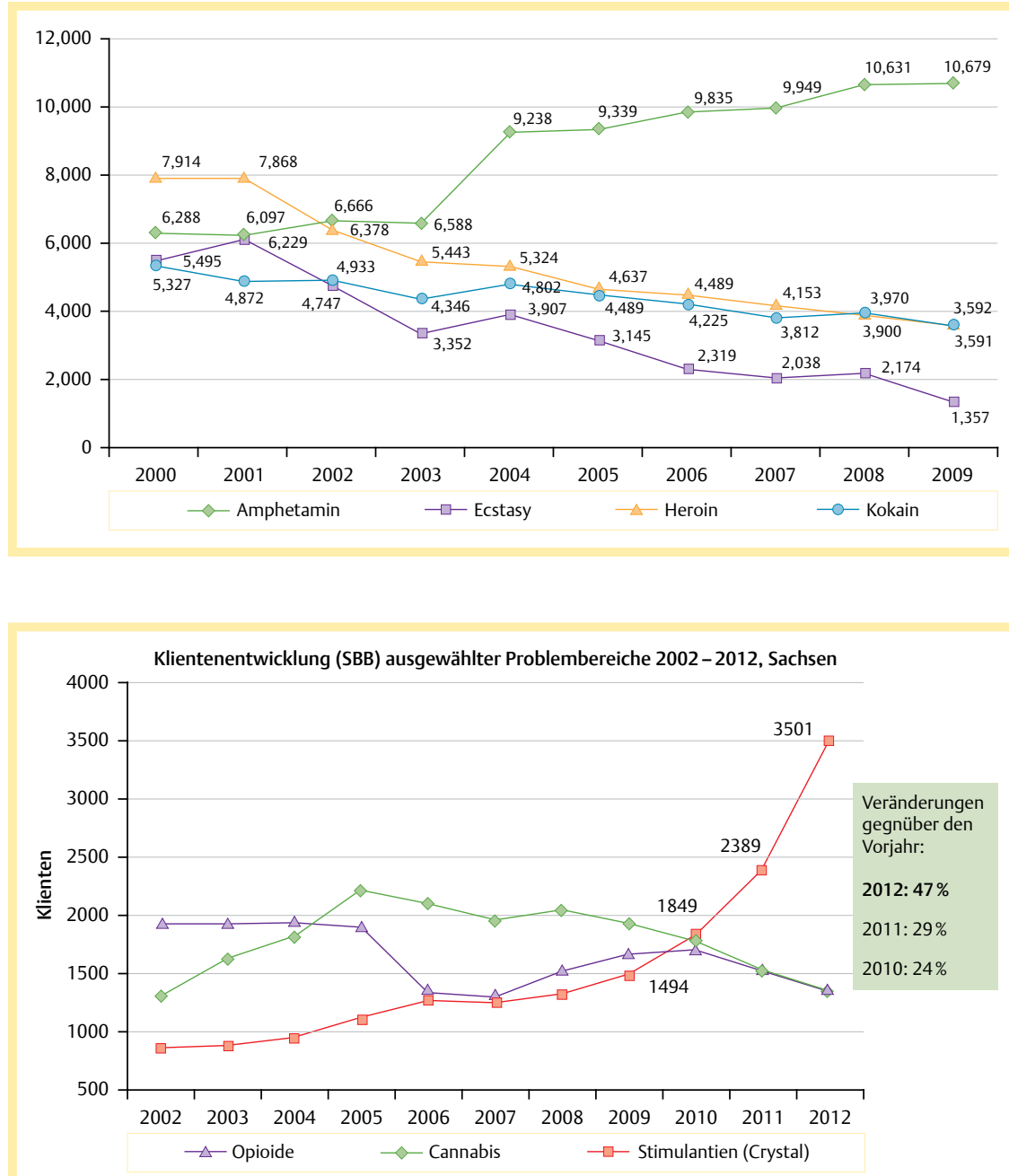

Abb. 1 Zunahme der ATS-Erstauffälligen seit 2000 aus ATS Global Assesment 2011.

Abb. 2 Zunahme der Behandlungsnachfragen wegen Stimulantien, Sächsische Landesstelle gegen die Suchtgefahren 2012 [6]. 


\section{Methamphetamin als Droge $\nabla$}

\section{Unterschiede zu d-amphetamin und dem Medikament Pervitin ${ }^{\circledR}$}

Methamphetamin (metamfetamin) und d-amphetamin wurden als Weckamine (Kaffeeersatz) und Appetitzügler sowie als Antidepressivas bereits vor dem 2. Weltkrieg in Dosierungen von 3-30 mg/Tag als Medikament (z. B. Pervitin ${ }^{\circledR}$ ) oral eingesetzt. Die klinischen Erfahrungen mit der raschen Toleranz- und Abhängigkeitsentwicklung sowie der Auslösung von Psychosen, auch in diesen „therapeutischen“ Dosierungen, führte in Deutschland bereits 1942 zur BTMG-Unterstellung. Heutzutage wird das kristalline Methamphetaminhydrochlorid (=Crystal-Meth/CrystalSpeed) bei dem ersten, meist nasalen Konsum, bereits in einer Dosis von fast $100 \mathrm{mg}$ (Wirkstoffgehalt $80-90 \%$ ) eingenommen, im Gegensatz zu dem „recreational“ Konsum von ca. 20 mg bei oralem -Konsum von „Speedpillen“ (d-amphetamin) in den 70er und 80er Jahre. Methamphetamin kann, im Gegensatz zu den meisten Phenetylaminen, wegen des geringen Siedepunktes verdampft und geraucht werden, mit ähnlich schnellem Wirkeintritt wie bei intravenösem Konsum.

\section{Wirkweise}

Die zentralen Wirkungen werden über eine starke indirekt dopaminerge (Aktivierung der Freisetzung, Hemmung der Wiederaufnahme), starke noradrenerge und geringergradige serotonerge Mechanismen vermittelt.

Verschiedene neuroadaptiven Prozesse wie up- and down Regulationen $[12,13]$ sind für die rasche Toleranzentwicklung bei wiederholtem Konsum verantwortlich [14]. Als Grund für eine raschere Abhängigkeitsentwicklung als z. B. bei Kokain [4] werden neben den deutlich höheren Dosierungen der reinen, kristallinen, nasal konsumierten Substanz die geringere Tachycardieneigung durch die höhere Lipophilie als z. B. beim d-amphetamin, postuliert. Eine noradrenerge periphere Wirkung (Tachycardie) wird meist als unangenehm erlebt und begrenzt sonst die Dosierung der ATS bei Drogenkonsumenten.

Methamphetamin wird in das präsynaptische Axon aufgenommen und wirkt dort über verschiedene Mechanismen neurotoxisch (s.u.). Die Toleranzentwicklung entwickelt sich für die verschiedenen Transmittersysteme und Wirkbereiche mit unterschiedlicher Geschwindigkeit. Die zur Erhaltung der Euphorie notwendigen hohen Dosierungen führen zu einer überreizten Wachheit, bei gleichzeitiger körperlicher Erschöpfung. Um Schlaf anzustoßen, entwickelt sich dann häufig ein polyvalentes Konsummmuster mit dem Übergang in Opiat-[15] und Benzodiazepinkonsum [16]. Auch THC wird dann „zum Runterkommen“ in hoher Dosierung als Selbstmedikation konsumiert, was die diagnostische Zuordnung der nicht selten auftretenden Psychosen erschwert.

Methamphetamin wird über Cytochrom P450 CYP2D6 per $\mathrm{N}$-Demethylierung zum Amphetamin verstoffwechselt und über die Niere ausgeschieden. Somit kann es mit herkömmlichen Drogentests nachgewiesen werden.

\section{Die Folgen}

Mit der Verdrängung des d-amphetamin durch Methamphetamin kam es in Australien zu einer Zunahme von Todesfällen wegen cardialer Komplikationen bzw. intracerebralen Blutungen [17] sowie zum gehäuften Auftreten von Amphetaminpsychosen [18]. Die Zunahme von Notfallaufnahmen in den US-Bundesstaaten wegen cardialer oder psychiatrischer Komplikationen war ein frühes Warnzeichen für die Ausbreitung von West nach Ost [19].
Frühe Überlegungen zu einer, bereits in den 80er Jahren bei der XTC-Forschung postulierten Auslösung einer Parkinsonerkrankung durch ATS-Konsum [20,21], wurden bestätigt [22,23].

Bei abstinenten Methamphetaminabhängigen wurden schon früh (bei Abstinenz rückbildungsfähige) neurokognitive Störungen nachgewiesen, mit Beeinträchtigung von Alltagstätigkeiten $[24,25]$. In Bildgebungsstudien war eine Zuordnung der funktionellen Einschränkungen zu strukturellen bzw. metabolischen Veränderungen bestimmter Hirnbereiche möglich, was mit der klinischen Erfahrung korreliert [26-29]. Trotz grundsätzlicher methodologischer Kritik an Bildgebungsstudien [30] dürfen diese Befunde als gesichert gelten. Auch die Erfahrung der Angehörigen, einer im Verlauf des Konsums zunehmenden Gefühlskälte mit Verlust einer Empathiefähigkeit -die psychiatrische Gutachter zur Fehldiagnose einer dissozialen Persönlichkeitsstörung verleiten kann- finden sich in ersten Untersuchungen plausible Korrelate $[31,32]$.

Die die Neurotoxizität begründenden Mechanismen einer retrograden Apoptose monoaminer Bahnen durch die Aufnahme des Methamphetamines in die präsynaptische Nervenzelle sind gut untersucht [33-35].

Der in den Medien gerne bemühte Methmouth konnte auch bei deutschen Patienten nachgewiesen werden [36,37], obgleich unsere Patienten, anders als in den USA, über eine Krankenversichertenkarte verfügen und die bei adrenerg bedingter Hyposalivation kariesbedingten Schäden im klinischen Alltag bereits zahnärztlich saniert und so weniger offensichtlich sind. Besonders betroffen sind hierbei die Kauflächen der Backenzähne durch den Bruxismus.

\section{Safer-Use-Überlegungen}

Mit Ethnologischer Methodik konnten in Deutschland verschiedene Subpopulationen mit höherer Konsumfrequenz herausgearbeitet werden [38]. Die größte und bekannteste Gruppe, in der bereits über Partydrogenprojekte Peergroup basierte Aufklärung betrieben wird, ist die Elektronische Dance Musik-Szene, früher Technoszene. Die Applikationsformen sind nicht nur bei Medikamenten, sondern auch beim Drogenkonsum relevant für Wirkstoffkonzentrationsspitzen und somit für die Neurotoxizität. Als relativ „safe use“ für Gelegenheitskonsumenten könnte nur ein niedrig dosierter oraler Konsum von z. B. $<20 \mathrm{mg}$ gelten. [39]. Dies würde die Substanz aber für die jetzigen Konsumenten, denen es auch um eine schnell einsetzende Euphorie geht, eher unattraktiv machen. Methamphetamin kann wegen des geringen Siedepunktes verdampft und geraucht werden. Diese, in einer Untersuchung [40] schneller zu Psychosen führende Konsumweise, war eine Zeitlang im Rahmen von safer-use-Projekten als Alternative zum i.V. Konsum wegen der geringeren HIV-Übertragungsrate propagiert worden. Eher sollten „rotzende" nasal konsumierende Gelegenheitskonsumenten zum niedrig dosierten oralen Konsum angehalten werden oder dazu, wenigstens immer ein eigenes Röhrchen zu verwenden und bei Sexualkontakten auf safer sex zu achten. Die Steigerung des sexuellen Antriebs erhöht das Risiko für gefährliches Sexualverhalten $[41,42]$, da wegen der substanzbedingten Schmerzunterdrückung, verletzungsträchtigere Sexualpraktiken zur Erreichung eines Höhepunktes notwendig werden. Die Durchseuchungsrate in Californien war unter Methusern höher als bei i.v. Opiatabhängigen [43]. Dies betrifft besonders die Homosexuellenszene [44]. Frühzeitig wurde in Europäischen Großstädten mit einer aktiven Darkroom-undergroundszene entsprechende Aufklärung angeboten [45-47]. 


\section{Klinische Konsequenzen}

$\nabla$

\section{Postkonsum- bzw. Entzugssymptome}

Durch Dopamin- Noradrenalin- und Serotoninverbrauch sind die Symptome zu erklären: Anhedonie, Antriebslosigkeit, psychomotorische Verlangsamung im Wechsel mit hyperkinetischen Phasen, ein generelles Schwächegefühl, gereizt- depressive Stimmung mit reduziertem Selbstwertgefühl und Suizidgedanken sowie Kopfschmerzen führen zum Craving und dem erneuten Konsum, um o.g. Symptome zu lindern. Im Entzug sind vegetative Entgleisungen kaum zu erwarten, aber suizidale Ideationen häufig und im Praxisalltag zu berücksichtigen. Gesteigertes Hungergefühl führt zu einer besonders Patientinnen belastenden Gewichtszunahme über das ursprüngliche Gewicht hinaus. In Entzug und Rehabilitation ist Ernährungsberatung zur Verhütung solcher, subjektiv nicht tolerierter Gewichtszunahme, bei dieser Klientel besonders sinnvoll.

Auch die Schlafstörungen sind Rückfallauslöser. Die Langzeitwirkung der Amphetamine auf den Schlaf sind seit den 60er Jahren gut beforscht [48]. Im Entzug kommt es zunächst zu einer „crashphase“ [49] mit vermehrtem Schlaf, nach wenigen Tagen zu einem REM- Rebound mit nächtlichem Erwachen und vermehrten Drogenträumen, die ein Risiko für Rückfälligkeit und Therapieabbrüche darstellen. Die Anhedonie und Antriebslosigkeit kann wegen der neuroadaptiven Prozesse und der Neurotoxizität über Monate bestehen bleiben. Die Patienten sind durch die, bei chronischem Konsum häufig zu bemerkenden kognitiven Störungen, beeinträchtigt. Überforderungserleben in der Alltagsbewältigung bzw. im stationären Setting sind erlebbare Rückfallgründe.

Bei Aufnahme auf einer Entzugsstation im intoxikierten Zustand ist das Team durch das hohe Redebedürfnis („Laberflash“), die motorische Unruhe und Umtriebigkeit gefordert. Ergotherapeutische Maßnahmen wie Mandalas ausmalen, die Übernahme von Tischputzdiensten sowie ein immer verfügbarer Kunst-Ergotherapieraum mit der „Erlaubnis“, verschiedenste Projekte zu beginnen und auch abzubrechen, sind Milieugestaltungsmaßnahmen, die nach unserer klinischen Erfahrung die Abbruchquote in dieser Phase des Entzuges reduzieren können. Teams von Drogenentzugsstationen sehen und spüren bei akuter Alkohol-, Benzodiazepin- oder Opioidintoxikationen, dass gegebene Informationen zu den Stationsabläufen usw. nicht gespeichert werden. Stimulantienuser erscheinen wach, focussiert und aufmerksam- nur leider sind sie „eingeschaltet“ auf ganz andere Dinge focussiert, z.B. auf die Anordnung der Sommersprossen im Gesicht des über die Stationsabläufe informierenden Pflegers. Sie haben wegen der oben beschriebenen kognitiven Störungen Mühe, sich selber den Alltag zu organisieren. Patienten im niedrigschwelligen stationären Entzug mit der Hauptsubstanz Methamphetamin kommen im Vergleich zu Opiatpatienten schneller zur Behandlung, waren rascher für weiterführende Behandlungen zu motivieren und schlossen die Behandlung häufiger regulär ab [50]. Therapiesettings sind an die hohe, teils primäre, teils sekundäre Comorbidität anzupassen [51].

\section{Psychosen}

Die Zunahme amphetamininduzierter Psychosen bei Auftreten des Methamphetamins in der Drogenszene wurde an verschiedenen Orten nachgewiesen [18,52]. Auftreten und Verlauf scheinen, wie die methamphetaminassoziierte Gewalttätigkeit, gesamtdosisabhängig zu sein. [53,54]. Überlegungen zu einer genetischen Vulnerabilität sowie die Fragen der Auslösung einer eigenständigen Erkrankung vs. der Auslösung einer Schizophre- nie (Vulnerabilitäts- Stressmodell) werden in den unterschiedlichen Psychiatriekulturen noch kontrovers diskutiert z.B. [55]. Bei der Behandlung der Methamphetaminassoziierten Psychosen (MAP) gibt es neben der klinischen Erfahrung auch Hinweise aus Tierversuchen, eher auf Haloperidol zu verzichten [56,57]. Bei längeren Wachphasen vor Beginn der Psychose kann der Schlafentzug auslösend sein. In der akutpsychotischen Phase erscheint eine kurzfristige Benzodiazepingabe auch deshalb klinisch hilfreich, jedoch fehlt hierzu wissenschaftliche Evidenz. Bei der Auswahl der Neuroleptika ist zu bedenken, dass abhängigen Konsumenten im Entzug nach eigenen Daten ohnehin mit einer mittleren Gewichtszunahme von ca $8 \mathrm{~kg}$ zu rechnen haben.

Patienten mit einer drogeninduzierten Psychose profitieren subjektiv von einem 3 stündigen Psychoedukationsprogramm, auch bereits auf einer beschützenden Station (nach Abklingen eines Erregungszustandes) [58].

Ein Ansäuern des Harns kann die Wirkungsdauer des Methamphetamins verringern.

\section{Beratung}

Für die ambulante Behandlung hat sich eine Terminvergabe innerhalb von $24 \mathrm{~h}$ bewährt. Amphetaminpatienten gelten im intoxikierten Zustand wegen des Verlustes des Zeitempfindens und der zwanghaft anmutenden Focussierung auf Banalitäten als wenig zuverlässig bezüglich Termineinhaltung. Moderne Kommunikationsmittel mit Erinnerungs-SMS sowie Erinnerungsanrufen scheinen nach Erfahrungen der bereits betroffenen Suchtberatungsstellen die Teilnahmequote zu verbessern. Selbstbehandlungsstrategien sind wirksam [59] und werden gegenwärtig in einem Onlineportal in Deutschland gefördert [60].

\section{Rehabilitation}

Aus den USA liegen verschiedene Studien zu den Psychotherapieergebnissen vor. Eine Haltequote von $43 \%$ und 69\% Punktabstinenz bei der 6-Monatskatamnese konnten im integrativen „Matrix“-Programm gezeigt werden [61-63]. Das Manual ist in der englischen Version frei verfügbar [64], eine autorisierte Übersetzung liegt bisher nicht vor. Sowohl kognitiv verhaltenstherapeutische und Community Reinforcement-Ansätze, als auch das 12-Schritte-Programm, erwiesen sich als wirksam [65]. Kontingenz-Management (CM) war bei dem, für die USA-typischen Sozialsystem, jedoch genauso hilfreich, um während der Studienzeit drogenfreie Urintests zu erreichen [66-68]. Aus Deutschland liegt bisher nur eine eigene unspezifische Untersuchung zum Verlauf nach stationärer Entgiftung vor [69].

\section{Medikamentöse Behandlungsstrategien}

In Metaanalysen blieben klassisch pharmakopsychiatrische Ansätze bisher nur von bescheidener Wirksamkeit für das Ziel der Abstinenz [70,71].

Die häufig eingesetzten SSRIs werden nur bei vordiagnostizierter Depression empfohlen, die Haltequote war bei unzureichender Wirkung gegen Antriebsstörung und Anhedonie geringer als bei Placebo, was mit den Nebenwirkungen begründet wurde. Die Anhedonie im Methamphetaminentzug sei nicht mit der Anhedonie bei Major Depression vergleichbar, was in Abwägung der hauptsächlichen dopaminerg vermittelten Wirkung einleuchtend ist [72]. Bupropion konnte in ersten Studien eine Wirksamkeit, allerdings auch nur bei nicht täglich konsumierenden Patienten, zeigen [73]. Naltrexon war gegenüber Placebo bezüglich Haltequote, Selbstangaben des Amphetaminkonsums und dies überprüfenden Drogenscreenings überlegen [74]. 
Dopaminanaloga/Amphetaminsubstitution?

Eine Reihe von Forschungsgruppen bemüht sich um die Dopamin-Analogabehandlung, d.h. Substitutionsbehandlung z.B. mit retardiertem Amphetaminsulfat [Übersicht 75]. Erst in höheren Dosierungen scheint dies neben schadensminimierenden Erfolgen, wie Einstellung des i.v.-Konsums [76] auch zu einer Beigebrauchsreduktion zu führen [77,78]. Methylphenidat (retardiert) zeigte in Studien bisher in den üblichen Dosierungen diesbezüglich keine ausreichende Wirksamkeit. Die Haltequote war gegenüber Placebo jedoch verbessert [79].

Zur Milderung der Entzugssymptome im stationären Entzug, ist Modafinil in ersten Studien hilfreich gewesen [80,81]. Untersuchungen zum, nach klinischer Erfahrung wirksamen, Koffein, fehlen.

Bei weiterer Ausbreitung des Methamphetaminproblems könnte eine Dopaminanalogabehandlung (z. B Substitution mit retardiertem Amphetaminsulfat bzw. stationärer fraktionierter homologer Entzug mit Modafinil) in betroffenen Großstädten Ziel von Forschungsvorhaben werden. Bei immer kürzer werdenden Behandlungszeiten im stationären qualifizierten Entzug, könnte die Rehabilitationsfähigkeit ebenfalls durch vorübergehende Dopaminanalogabehandlung [82] verbessert werden.

\section{Fazit für die Praxis}

Es ist unwahrscheinlich, dass Zentraleuropa vom weltweiten Trend zu kristallinen Methamphetamin ausgenommen bleibt. Unter Kenntnis der höheren Wirksamkeit der nasal, hochrein eingenommenen Substanz sind spezifische safer use- und Präventionstrategien für Subpopulationen zu entwickeln. Die Behandlungsergebnisse aus anderen Ländern sind ermutigend, müssen aber auf den deutschen Sprachraum übertragen werden. Medikamentöse Strategien sind noch unzureichend beforscht. Für eine Analogabehandlung mit d-amphetamin (Amphetaminsubstitution) besteht in Deutschland wegen den notwendigen großen Verordnungsmengen nach der BTMVV weder eine Rechtsgrundlage, noch eine legitimierende Datenlage. Die Gefahr der Neurotoxizität, im Gegensatz zur Opiatsubstitution, muss bei der Planung von Amphetaminsubstitutionsstudien genau abgewogen werden.

\section{Zur Person}

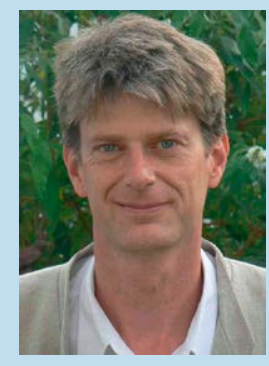

Dr. Härtel Petri war langjähriger Leiter der Fachklinik Hochstadt sowie des Suchtbereiches am Bezirkskrankenhaus Bayreuth. Durch die Nähe zur Tschechischen Republik musste sich sein Team ab 1997 auf die sich freiwillig und selbstmotiviert zum qualifizierten Entzug einfindenden, aber auch auf die notfallmäßig eingelieferten intoxierten Patienten einstellen und die Behandlungssettings nach angelsächsischem Vorbild anpassen. Als Leiter einer Substitutionsambulanz und in persönlicher Kenntnis der Amphetaminsubstitution in Großbritannien sieht er deren wissenschaftlich begleitete Implementierung als zukünftig notwendig an, sofern der Trend zum Methamphetamin bestehen bleibt. Er ist mittlerweile in eigener psychotherapeutisch-psychiatrischer Praxis niedergelassen.
Interessenkonflikt: Der Autor gibt an, dass kein Interessenkonflikt besteht.

\section{Literatur}

12011 Global ATS Assessment UNODOC, United Nations Publication Sales.No.E.11.XI.13). Seite 54 http://www.unodc.org/documents/ATS/ ATS_Global_Assessment_2011.pdf

2 Anglin MD, Burke CPerrochet et al. History of the methamphetamine problem. J Psychoactive Drugs 2000; 3: 137-141

3 Kraus L, Pabst A, Piontek $D$ et al. Substanzkonsum und substanzbezogene Störungen: Trends in Deutschland 1980-2012. Sucht 2013; 59: 333-345

4 Gonzalez CF, Barrington EH, Walton MA et al. Cocaine and methamphetamine: differential addiction rates. Psychol Addict Behav 2000; 14: 390-396

5 Kalechstein $A D$, Newton TF, Longshore D et al. Psychiatric comorbidity of methamphetamine dependence in a forensic sample. J Neuropsychiatry Clin Neurosci 2000; 12: 480-484

6 Sucht 2012: Bericht der Suchtkrankenhilfe in Sachsen, März 2013 http://www.slsev.de/Sucht2012.pdf

7 Härtel-Petri R, Schulthei $\beta$ P, Wolfersdorf $M$. Stimulantienabhängigkeit in Oberfranken weiter zunehmend: Zunahme der Abhängigen von „Crystal-Speed“ 1996-1999. Nervenarzt 2001; 73 S1: 151

8 Piontek D, Pfeiffer-Gerschel T, Jakob L et al. Sachbericht Sekundäranalysen im Rahmen des BMG-Projekts „Missbrauch von Amphetaminen in Deutschland: Studie zur Motivation und zu den Konsumgewohnheiten von missbräuchlich Amphetaminkonsumierenden. 2014; Online: http://www.drogenbeauftragte.de/fileadmin/dateien-dba/Drogen undSucht/Illegale_Drogen/Heroin_andere/Downloads/2014-05-21_ Sachbericht-Projekts_Missbrauch_von_Amphetaminen_.pdf

9 http://www1.wdr.de/fernsehen/aks/themen/Apaan100.html

10 http://www.ndr.de/regional/zoll483.html

11 http://www.stern.de/panorama/crystal-meth-neonazi-drogen ring-in-leipzig-vor-gericht-1974896.html

12 Castner SA, Goldman-Rakic F. Long-lasting psychotomimetic consequences of repeated low-dose amphetamine exposure in rhesus monkeys. Neuropsychopharmacology 1998; 20: 10-28

13 White FJ, Kalivas PW. Neuroadaptations involved in amphetamine and cocaine addiction. Drug Alcohol Dep 1998; 51: 141-153

14 Wilens TE, Spencer TJ. Pharmacology of Amphetamines. In: Tarter RE, Ammerman RT, (Hrsg.). Handbook of substance abuse. New York: Plenum Press; 1998: 501-513

15 Hando J, Hall W. Patterns of amphetamine use in Australia. In: Klee H, (Hrsg.). Amphetamine Misuse. International Perspectives on Current Trends. Amsterdam: Overseas Publishers Association; 1997: 81-97

16 Darke S, Cohen J, Ross J.et. al. Transitions between routes of administration of regular amphetamine users. Addiction 1994; 89: 1077-1083

17 Kaye S, Darke S, Duflou J et al. Methamphetamine related fatalities in Australia: demographics, circumstances, toxicology and major organ pathology. Addiction 2008; 103: 1353-1360

18 Australian Institute of Health and Welfare. AIHW National Hospital Morbidity Database. Separation, patient day and average length of stay statistics by principal diagnosis in ICD-10-AM, Australia, 1998-99 to 2003-04. http://www.aihw.gov.au/cognos/cgi-bin/ppdscgi.exe?DC = $\mathrm{Q} \& \mathrm{E}=$ /AHS/principaldiagnosis0304 (accessed Dec 2006) zit. nach Gordian W, Fulde O, Wodak A. Ice: cool drug or real problem? MJA 2007; 186: 334-335

19 NIDA Notes. 1998;PDF- http://www.drugabuse.gov/sites/default/files/ rrmetham.pdf

20 Guilarte TR. Is methamphetamine abuse a risk factor in Parkinsonism? Neurotoxicology 2001; 22: 725-731

21 Ricaurte G, Mechan AO, Yuan J et al. Amphetamine treatment similar to that used in the treatment of adult attention-deficit/hyperactivity disorder damages dopaminergic nerve endings in the striatum of adult nonhuman primates. J Pharmacol Exper Therap 2005; 315: 91-98

22 Garwood ER, Bekele W, McCulloch CE et al. Amphetamine exposure is elevated in Parkinson's disease. Neurotoxicology 2006; 27: 1003-1006

23 Callaghan RC, Cunningham JK, Sykes J et al. Increased risk of Parkinson's disease in individuals hospitalized with conditions related to the use of methamphetamine or other amphetamine-type drugs. Drug Alcohol Depend 2012; 120: 35-40

24 Brook HL, Minassian A, Perry W. Effect of Methamphetamine dependence on everyday functional ability. Addict Behav 2010; 35: 593-598 
25 Volkow ND, Chang L, Wang GJ et al. Loss of dopamine transporters in methamphetamine abusers recovers with protracted abstinence. J Neurosci 2001; 21: 9414-9418

26 Kalechstein AD, Newton TF, Green M. Methamphetamine dependence is associated with neurocognitive impairment in the initial phases of abstinence. J Neuropsychiatry Clin Neurosci 2003; 15: 215-220

27 Ernst T, Chang L, Leonedo-Yee $M$ et al. Evidence for long-term neurotoxicity associated with methamphetamine abuse: A 1H MRS study. Neurology 2000; 54: 1344-1349

28 Hoffman WF, Moore M, Templin $R$ et al. Neuropsychological function and delay discounting in methamphetamine-dependent individuals. Psychopharmacology 2006; 188: 162-170

29 Johanson CE, Frey KA, Lundahl LH et al. Cognitive function and nigrostriatal markers in abstinent methamphetamine abusers. Psychopharmacology 2006; 185: 327-338

30 Hart CL, Marvin CB, Silver $R$ et al. Is cognitive functioning impaired in methamphetamine users? A critical review. Neuropsychopharmacology 2012; 37: 586-608

31 Sekine $Y$, Ouchi $Y$, Takei $N$ et al. Brain serotonin transporter density and aggression in abstinent methamphetamine abusers. Arch Gen Psychiatry 2006; 63: 90-100

32 Payer DE, Lieberman MD, London ED. Neural correlates of affect processing and aggression in methamphetamine dependence. Arch Gen Psychiatry 2011; 68: 271-282

33 Gouzoulis-Mayfrank E, Daumann J. Neurotoxicity of drugs of abuse - the case of methylenedioxy amphetamines (MDMA, ecstasy), and amphetamines. Dialog Clin Neurosci 2009; 11: 305-317

$34 \mathrm{Yu} S$, Zhu L, Shen $Q$ et al. Recent Advances in Methamphetamine Neurotoxicity Mechanisms and Its Molecular Pathophysiology. Behav Neurol 2015; in Print- Epub 2015 Mar 12: http://dx.doi. org/10.1155/2015/103969

35 Yamamoto BK, Moszczynska A, Gudelsky GA. Amphetamine toxicities: classical and emerging mechanisms. Ann NY Acad Sc 2010; 1187: 101-121

36 Boyer EM, Thompson N, Hill T et al. The relationship between Methamphetamine use and dental caries and missing teeth. J Dent Hyg 2015; 89: 119-131

37 Rommel N, Rohleder NH, Härtel-Petri R et al. Orale Manifestationen bei Methamphetamin-Abhängigkeit. Zahnärztliche Mitteilungen 2013 103: $34-38$

38 Milin S, Lotzin A, Degkwitz P et al. Amphetamin und Methamphetamin - Personengruppen mit missbräuchlichem Konsum und Ansatzpunkte für präventive Maßnahmen. Hamburg: Zentrum für Interdisziplinäre Suchtforschung (ZIS); 2014

39 Pates RM, Mitchell A. Amphetamine use in South Glamorgan. J Substance Misuse 1996; 1: 165-173

40 Matsumoto T, Kamijo A, Miyakawa T et al. Methamphetamine in Japan: the consequences of methamphetamine abuse as a function of route of administration. Addiction 2002; 97: 809-817

41 Sattah MV, Supawitkul S, Dondero TJ et al. Prevalence of and risk factors of methamphetamine use in northern Thai youths: results of an audio-computerassisted self-interviewing survey with urine testing. Addiction 2002; 97: 801-808

42 Semple S, Patterson TL, Grant I. Determinants of condom use among heterosexually-identified methamphetamine users. AIDS and Behaviour 2004; 8: 391-400

43 Anderson $R$, Flynn $N$. The Metamphetamine-HIV-Connection in Northern California. In: Klee H, (Hrsg.). Amphetamine Misuse, international Perspectives on current Trends. Overseas Publishers Association; Amsterdam: 1997: 181-197

44 Shoptaw S, Reback CJ. Methamphetamine use and infectious disease-related behaviours in men who have sex with men: implications for interventions. Addiction 2007; 102 (Suppl 1): 130-135

45 Mayer KH, Skeer MR, O'Cleirigh C et al. Factors associated with amplified HIV transmission behavior among American men who have sex with men engaged in care: implications for clinical providers. Ann Behav Med 2014; 47: 165-171

46 Thu Vu NT, Maher L et al. Amphetamine-type stimulants and HIV infection among men who have sex with men: implications on HIV research and prevention from a systematic review and meta-analysis. J Int AIDS Soc 2015; 18: 19-27

47 http://www.lifeormeth.com/london-i/4510297636

48 Baekeland F. Pentobarbital and dextroamphetamine sulfate: effects on the sleep cycle in man. Psychopharmacologia 1967; 11: 388-396

49 McGregor C, Srisurapanont M, Jittiwutikarn J et al. The nature, time course and severity of methamphetamine withdrawal. Addiction 2005; 100: 1320-1329

50 Rodler $R$, Härtel-Petri R, Steinmann J et al. Qualifizierte stationäre Drogenentzugsbehandlung. Krankenhauspsychiatrie 2006; 17: 15-18
51 Schäfer I, Lotzin A, Milin S. Ungedeckte psychotherapeutische Bedarfe bei Stimulanzienkonsumenten. Bedeutung komorbider Störungen und traumatischer Erfahrungen. Psychotherapeut 2014; 59: 300-305

52 Härtel-Petri $R$, Rodler $R$, Schmeisser $U$ et al. Increasing prevalence of amphetamine - and methamphetamine-induced psychosis. Psychiat Prax 2005; 32: 13-17

53 McKetin R, Lubman DI, Baker AL et al. Dose-related psychotic symptoms in chronic methamphetamine users: evidence from a prospective longitudinal study. JAMA Psychiatry 2013; 70: 319-324

54 McKetin R, Lubman DI, Najman JM et al. Does methamphetamine use increase violent behaviour? Evidence from a prospective longitudinal study. Addiction 2014; 109: 798-806

55 Bramness JG, Gundersen OJ, Guterstam J et al. Amphetamine-induced psychosis - seperate diagnostic entity or primary psychosis triggered in the vulnerable? BMC Psychiatry 2012; 12: 221

56 Hatzipetros T, Raudensky JG, Soghomonian JJ et al. Haloperidol treatment after high-dose methamphetamine administration is excitotoxic to GABA cells in the substantia nigra pars reticulata. Neuroscience 2007; 30; 27: 895-902

57 Verachai V, Rukngan W, Chawanakrasaesin $\mathrm{K}$ et al. Treatment of methamphetamine-induced psychosis: a double-blind randomized controlled trial comparing haloperidol and quetiapine. Psychopharmacology (Berl) 2014; 231: 3099-3108

58 Schmeisser U, Härtel-Petri R, Steinmann J et al. Psychoedukation bei Sucht und Psychose. Psychiatr Prax 2007; 34: 52-54

59 Wunderlich $R$, Härtel-Petri $R$, Rodler $R$ et al. Selbstbehandlungsversuche methamphetaminabhängiger Patienten - Längerfristige Katamnese stationär entgifteter Patienten. Nervenheilkunde 2008; 27 (Suppl 11a): 34-35

60 https://breaking-meth.de

61 Obert JL, McCann MJ, Marinelli-Casey $P$ et al. The matrix model of outpatient stimulant abuse treatment: history and description. J Psychoactive Drugs 2000; 32: 157-164

62 Shoptaw S, Rawson RAMcCann et al. The Matrix Model of outpatient stimulant abuse treatment: Evidence of Efficacy. J Addict Dis 1994; 129-141

63 Rawson RA, Marinelli-Casey PAnglin et al. A multi-site comparison of psychosocial approaches for the treatment of methamphetamine dependence. Addiction 2004; 99: 708-717

64 http://store.samhsa.gov/product/Matrix-Intensive-Outpatient-Treat ment-for-People-With-Stimulant-Use-Disorders-Counselor-s-Treat ment-Manual/SMA13-4152

65 Nowinski J, Baker S, Carroll K. Twelve Step Facilitation Therapy Manual: A Clinical Research Guide for Therapists Treating Individuals with Alcohol Abuse and Dependence. Rockville, MD: National Institute an Alcohol Abuse and Alcoholism; 1992

66 Rawson RA, McCann MJFlammino et al. A comparison of contingency management and cognitive-behavioral approaches for stimulant-dependent individuals. Addiction 2006; 101: 267-274

67 Roll JM, Petry NM, Stitzer ML et al. Contingency management for the treatment of methamphetamine use disorders. Am J Psychiatr 2006; 163: 1993-1999

68 Knapp WP, Soares B, Farrell $M$ et al. Psychosocial interventions for cocaine and psychostimulant amphetamines related disorders. Cochrane Database of Systematic Reviews 2007; 3: CD003023

69 Datzer S, Härtel-Petri R, Schiller $M$ et al. Rückfallrate metamphetaminabhängiger PatientInnen nach niedrigschwelligem Drogenentzug - Ergebnisse einer mittelfristigen Katamnese. Suchttherapie 2002; 3: 48-51

70 Karila L, Weinstein A, Aubin $\mathrm{H}-\mathrm{J}$ et al. Pharmacological approaches to methamphetamine dependence: a focused review. Br J Clin Pharmacol 2010; 69: 578-592

71 Brackins T, Brahm NC, Kissack JC. Treatments for methamphetamine abuse: a literature review for the clinician. J Pharm Pract 2011; 24: 541-550

72 Shoptaw S, Huber A, Peck JY et al. Randomized, placebo-controlled trial of sertraline and contingency management for the treatment of methamphetamine dependence. Drug Alcohol Depend 2006; 85: 12-18

73 Elkashef AM, Rawson RA, Anderson AL et al. Bupropion for the treatment of methamphetamine dependence. Neuropsychopharmacology 2008: 33: 1162-1170

74 Jayaram-Lindström $N$, Hammarberg A, Beck $O$ et al. Naltrexone for the treatment of amphetamine dependence: a randomized, placebo-controlled trial. Am J Psychiatry 2008; 165: 1442-1448

75 Pérez-Mañá C, Castells X, Torrens $M$ et al. Efficacy of psychostimulant drugs for amphetamine abuse or dependence. Cochrane Database of Systematic Reviews 2013; 9: CD009695

76 Charnaud $B$, Griffith $V$. Levels of intravenous drug misuse among clients prescribed oral dexamphetamine or oral methadon: a comparison. Drug Alcohol Depend 1998; 52: 79-84 
77 Galloway GP, Buscemi R, Coyle JR et al. A randomized, placebo-controlled trial of sustained-release dextroamphetamine for treatment of methamphetamine addiction. Clin Pharmacol Ther 2011; 89: 276-282

78 Longo $M$, Wickes $W$, Smout $M$ et al. Randomized controlled trial of dexamphetamine maintenance for the treatment of methamphetamine dependence. Addiction 2010; 105: 146-154

79 Miles SW, Sheridan J, Russell B et al. Extended-release methylphenidate for treatment of amphetamine/methamphetamine dependence: a randomized, double-blind, placebo-controlled trial. Addiction 2013; 108: $1279-1286$
80 McGregor C, Srisurapanont M, Mitchell A et al. Symptoms and sleep patterns during inpatient treatment of methamphetamine withdrawal: a comparison of mirtazapine and modafinil with treatment as usual. J Subst Abuse Treat 2008; 35: 334-342

81 Hester $R$, Lee $N$, Pennay A et al. The effects of modafinil treatment on neuropsychological and attentional bias performance during 7-day inpatient withdrawal from methamphetamine dependence. Exp Clin Psychopharmacol 2010; 18: 489-497

82 Anderson AL, Li SH, Biswas $K$ et al. Modafinil for the treatment of methamphetamine dependence. Drug Alcohol Depend 2012; 120: 135-141 